\title{
Féeries
}

Études sur le conte merveilleux, XVII $-\mathrm{XIX}{ }^{\mathrm{e}}$ siècle

\section{Ibrahim et Anaïs : le merveilleux comme expérience du droit des femmes au plaisir dans les Lettres persanes (1721) de Montesquieu}

The story "Ibrahim and Anaïs": the supernatural as an experience of women's right to pleasure in Montesquieu's Lettres persanes (1721)

\section{Moussa Traoré}

\section{OpenEdition}

Journals

Édition électronique

URL : http://journals.openedition.org/feeries/1517

DOI : $10.4000 /$ feeries. 1517

ISSN : 1957-7753

Éditeur

UGA Éditions/Université Grenoble Alpes

Édition imprimée

ISBN : 978-2-37747-075-4

ISSN : $1766-2842$

\section{Référence électronique}

Moussa Traoré, «Ibrahim et Anaïs : le merveilleux comme expérience du droit des femmes au plaisir dans les Lettres persanes (1721) de Montesquieu », Féeries [En ligne], 15 | 2018, mis en ligne le 22 janvier 2019, consulté le 21 décembre 2020. URL : http://journals.openedition.org/feeries/1517 ; DOI : https://doi.org/10.4000/feeries. 1517

Ce document a été généré automatiquement le 21 décembre 2020.

(c) Féeries 


\title{
Ibrahim et Anaïs : le merveilleux comme expérience du droit des femmes au plaisir dans les Lettres persanes (1721) de Montesquieu
}

\author{
The story "Ibrahim and Anaïs": the supernatural as an experience of women's \\ right to pleasure in Montesquieu's Lettres persanes (1721)
}

Moussa Traoré

L'univers sensible du conte merveilleux oriental a souvent servi de cadre aux auteurs des Lumières pour réfléchir à la condition des femmes et à leur bonheur dans une société sous domination masculine. Le conte Ibrahim et Anaïs, inséré dans les Lettres persanes (1721) de Montesquieu, s'inscrit en grande partie dans cette problématique. Il constitue une fiction heuristique dans laquelle est menée une réflexion sérieuse sur le droit des femmes au plaisir comme condition d'un retour à un ordre social plus harmonieux, où le voir et le sentir tendent à suppléer le savoir et le pouvoir. L'analyse d'Aurélia Gaillard a beaucoup contribué à la prise en compte de ce conte enchâssé dans la lettre 141 comme "un instrument à penser le renversement interprétatif : une expérimentation du retournement ${ }^{1} »$. En poussant l'analyse, nous essaierons de lire ce texte non plus comme l'expérimentation du monde inversé, mais comme la représentation des aberrations d'une société qui réprime le désir féminin et la figuration d'un retour à un société plus conforme à la Nature ${ }^{2}$ parce que libératrice des sens des femmes. Si nous partons du postulat que dans l'anthropologie de Montesquieu, l'être humain est posé en tant que corps, sensibilité et intelligence (Livre I de L'Esprit des lois), et que la nature sensible et sensuelle ${ }^{3}$ des hommes et des femmes aspire irrésistiblement au plaisir, qu'en est-il des femmes abandonnées et vivant dans la privation? Un rapport social des sexes plus apaisé n'appelle-t-il pas à dépasser la représentation masculine des femmes incarnant un idéal de disponibilité totale, portées à faire plaisir sans jamais voir leurs désirs assouvis? 
1 Dans notre étude qui sera à la fois littéraire, philosophique et socio-anthropologique, il s'agira de montrer comment l'univers sensible du conte merveilleux Ibrahim et Anaïs participe à la réflexion sur la gestion de la sexualité féminine dans les sociétés souvent dominées par les hommes, et donne une réponse à la question du rapport social conflictuel entre les deux sexes. Si dans les sociétés patriarcales la femme est condamnée à ne satisfaire que les désirs de l'homme, le conte Ibrahim et Anaïs fait de la réhabilitation du plaisir féminin la condition d'une société harmonieuse et pérenne. Ce conte merveilleux semble donc montrer comment la répression du désir féminin devient un facteur de déséquilibre pour la société et comment la libération de la sensualité féminine peut devenir le gage d'une harmonie sociale.

\section{La répression des plaisirs féminins}

L'enfermement des femmes liée à l'idée de leur infériorité physique et morale ${ }^{4}$ par la société musulmane persane - et en filigrane par la société européenne encore très chrétienne au XVIII ${ }^{\mathrm{e}}$ siècle - se double d'une répression de leur désir comme seul gage d'une organisation sociale vertueuse. Car, dans l'imaginaire des trois grandes religions monothéistes (Judaïsme, Christianisme et Islam), la femme est un être dont les désirs excessifs doivent être contrôlés et orientés pour satisfaire ceux de l'homme. En effet, ces religions monothéistes voient la femme comme la figure par excellence de la tentation, dont les ardeurs insatiables risquent de troubler l'ordre social. Du Moyen Âge au XVIII ${ }^{\mathrm{e}}$ siècle, la femme est souvent perçue comme, par nature, malhonnête du fait de ce sexe indomptable et indompté. C'est donc à l'époque des Lumières, à l'époque du libertinage, que va se réaliser ce vieux fantasme masculin de pouvoir contrôler la sensualité des femmes.

La gestion du désir féminin dans le sérail d'Ibrahim reprend à son compte la conception restrictive des plaisirs des femmes comme seul moyen d'éviter un désordre moral dans la société. Cet univers clos fonctionne comme une société despotique qui refuse tout plaisir aux femmes, condamnées à ne satisfaire que les désirs de leur maître. Les circonstances de la mort d'Anaïs - qui tiennent lieu de scène d'exposition - montrent comment l'enfermement des femmes obéit, chez Ibrahim, à une logique de domination masculine contre-nature, fondée sur la privation de plaisirs. Ibrahim incarne le prototype du seigneur persan qui enferme un nombre important de femmes dans un sérail.

Un homme, nommé Ibrahim, était d'une jalousie insupportable. Il avait douze femmes extrêmement belles, qu'il traitait d'une manière très dure : il ne se fiait plus à ses eunuques, ni aux murs de son sérail ; il les tenait presque toujours sous la clef, enfermées dans la chambre, sans qu'elles pussent se voir, ni se parler; car il était même jaloux d'une amitié innocente : toutes ses actions prenaient la teinture de sa brutalité naturelle : jamais une douce parole ne sortit de sa bouche; et jamais il ne fit le moindre signe, qui n'ajoutât quelque chose à la rigueur de leur esclavage ${ }^{5}$. Le sérail du vrai Ibrahim favoriserait donc la recomposition d'une société despotique ${ }^{6}$ et contre-nature, puisque fonctionnant sous le principe de la contrainte, de la crainte et de la privation des plaisirs. Pour comprendre cette présentation sommaire de la condition servile des femmes d'Ibrahim et de la privation des plaisirs sensuels dont elles sont victimes, il faut lire le conte comme prémonition et comme réponse à la situation des femmes dans le sérail d'Usbek, lieu de claustration qui est sous la menace d'une désagrégation. D'ailleurs, le traitement réservé aux femmes au début du conte 
Ibrahim et Anaïs renvoie à celui qu'Usbek inflige aux siennes: les femmes sont des prisonnières gardées par des eunuques en vue de satisfaire les désirs d'un seul homme puissant et injuste.

Lieu clos et étroitement surveillé par de nombreux eunuques, le sérail constitue une prison $^{7}$ luxueuse dans laquelle languissent les épouses d'Usbek. C'est ce que suggèrent les lettres 3 et 7, adressées par Zachi puis Fatmé à Usbek: la situation d'énonciation permet aux deux femmes d'exprimer librement leur amour et leurs griefs. Ne vivant que pour plaire à leur maître, elles sont d'abord livrées à un désir physique qui les torture dès lors que leur époux s'est absenté. Zachi évoque ainsi la douleur de parcourir " ces lieux qui, me rappelant sans cesse mes plaisirs passés, irritaient tous les jours mes désirs avec une nouvelle violence ${ }^{8}$ ", au moment où Fatmé avoue que "[son] imagination se perd dans ses désirs, comme elle se flatte dans ses espérances ${ }^{9}$ ». La frustration semble être le sentiment qui domine chez toutes ces femmes. Zachi l'exprime en décrivant les manifestations physiques de ce manque ( Je pousse des soupirs qui ne sont point entendus ; mes larmes coulent, et tu n'en jouis pas $\left.{ }^{10} »\right)$, tandis que Fatmé utilise la métaphore précieuse du feu qui la consume: "[...] le feu, qui me dévore, dissipe lui-même ces enchantements et rappelle mes esprits. Je me trouve pour lors si animé... [...] et il est impossible de vivre dans cet état; le feu coule dans mes veines $[. . .]^{11}$.» L'aliénation des épouses résulte, par ailleurs, de l'obligation de se consacrer exclusivement à l'agrément de leur maître, y compris quand il est absent. Fatmé exprime ce paradoxe quand elle écrit :

Ne pense pas que ton absence m'ait fait négliger une beauté qui t'est chère. Quoique je ne doive être vue de personne, et que les ornements dont je me pare soient inutiles à ton bonheur, je cherche cependant à m'entretenir dans l'habitude de te plaire: je ne me couche point que je ne me sois parfumée des essences les plus délicieuses ${ }^{12}$.

Si l'aspiration aux plaisirs est inscrite en l'être humain comme un besoin élémentaire, et plus encore comme un droit $^{13}$, il est à noter que la loi du désir obsède les femmes du sérail. Les femmes d'Usbek, pour qui le bonheur est généralement à chercher dans la satisfaction des sens, éprouvent jusque dans leur chair les conséquences de la frustration sexuelle. Usbek et Ibrahim, cependant, ne peuvent accepter la réalisation réglée de tous les désirs et travaillent à éradiquer les plaisirs sensuels, à purifier le corps féminin de ses pulsions jugées infâmes par l'ascèse de la terreur. Le sérail des Lettres persanes est un lieu romanesque du féminin où les rapports entre les sexes sont pensés en termes de rapports de force qui penchent souvent en faveur de l'homme. En effet, la claustration des femmes dans le sérail traduit un idéal de répression de la libido féminine qui peut s'expliquer par la crainte de « la servitude de l'homme à l'égard des sens, donc des femmes ${ }^{14}$ ». C'est d'ailleurs l'idée avancée par Christophe Martin ${ }^{15}$ selon laquelle les espaces du féminin (le sérail, le boudoir, le couvent) sont des lieux marqués par rapport de force et d'une pulsion d'emprise qui prend essentiellement deux formes dans le roman du XVIII siècle : le fantasme d'une effraction dans l'intimité de la femme et celui d'une claustration du corps féminin. Cette pulsion ne se conçoit guère sans une hantise secrète que ces textes mettent en lumière: la crainte d'un empire féminin faisant peser sur les hommes une menace de féminisation, d'où la nécessité d'une réclusion domestique des femmes et d'une nouvelle économie des relations entre les sexes.

Dans ce « rapport social des sexes ${ }^{16}$ ", Montesquieu met en évidence l'omniprésence et la permanence de la domination masculine dans la société orientale représentée dans le conte, à laquelle la toute-puissance de l'homme dans la société occidentale, pourtant 
considérée comme plus civilisée, n'a rien à envier. Qu'il s'agisse du personnage oriental (Usbek et Ibrahim) qui se voit déposséder de tout pouvoir sur l'espace politique despotique, ou de l'homme occidental à qui s'adresse le récit et qui vit une période de crise politique, économique et religieuse, le besoin de resserrer le contrôle sur les femmes, notamment sur leur sexualité, fonctionne comme un substitut à leur pouvoir perdu. L'oppression des femmes à travers leur corps et leur sexe constitue le seul moyen pour l'homme d'affirmer sa virilité et son pouvoir quand les socles profonds de la société s'effondrent. La multiplicité des femmes, qui empêche la fixation sur un seul objet, et la scène d'élection de l'une parmi toutes les autres au sein du sérail (Lettre 3) manifestent la supériorité du despote et font de la privation sexuelle un instrument de domination. Dans cette microsociété qu'est le sérail, la femme est vouée à calmer les ardeurs sensuelles de l'homme, sans jamais accéder au repos des plaisirs assouvis. C'est ce que résume parfaitement Jean Goldzink en ces termes :

La femme est condamnée par la nature à libérer le corps des hommes et à souffrir perpétuellement de passions inassouvies que sa structure organique interdit de calmer. Elle a donc été conçue pour l'utilité du sexe masculin, et avant tout, apparemment, pour le délivrer des passions ${ }^{17}$.

Cette privation de plaisirs dont souffrent les femmes et entretenue dans les sérails d'Ibrahim et d'Usbek est analysée par Montesquieu comme l'incapacité d'une société patriarcale à trouver une réponse juste à la lancinante question de savoir que faire du désir féminin. La réponse d'Ibrahim, qui est une caricature de celle d'Usbek, constitue un viol du droit naturel ${ }^{18}$ qui fait de la satisfaction des sens un besoin vital. Cette aberration de la nature que constitue la répression du désir féminin n'est pas sans conséquence car elle participe à la dépopulation. La fécondité, qui est une loi de propagation de l'espèce humaine, est alors contrariée par la famine sexuelle dans laquelle se trouvent les femmes du sérail. Usbek n'a réussi à avoir qu'un enfant avec ses cinq femmes, alors qu'Ibrahim n'en a aucun avec ses douze épouses.

4 La frustration sexuelle qui caractérise le sérail représente une situation contre-nature qui engendre la corruption des mœurs et la révolte contre l'ordre de ce lieu clos. Cette insatisfaction sensuelle des femmes, soigneusement entretenue, appelle fatalement des plaisirs substitutifs, que Diderot illustrera en contexte chrétien dans La Religieuse ${ }^{19}$. Les plaintes de Zéphis, l'une des épouses d'Usbek, mettent en lumière cette recherche d'une volupté substitutive à celle frustrée par l'absence de son époux :

Enfin ce monstre noir a résolu de me désespérer. Il veut, à toute force, m'ôter mon esclave Zélide, Zélide qui me sert avec tant d'affection, et dont les adroites mains portent partout les ornements et les grâces. Il ne suffit pas que cette séparation soit douloureuse; il veut encore qu'elle soit déshonorante. Le traître veut regarder comme criminels les motifs de ma confiance $[. . .]^{20}$.

La Lettre 53 tourne autour du même motif. Le lecteur a droit à des considérations sur les possibles compensations sensuelles quand un eunuque blanc veut épouser une esclave de Zélis (Zélide). Le sort malheureux qui attend Zélide auprès de son mari châtré ressemble fort étrangement à celui des épouses d'Usbek alors absent depuis deux ans environ. À l'image de Zélis, Roxane, l'épouse favorite d'Usbek, a trouvé des moyens substitutifs de se faire plaisir au sein du sérail à travers une soumission feinte et en recevant son amant dans son appartement. Roxane pose son droit inaliénable au plaisir sensuel en le justifiant ainsi en nature: «J'ai réformé tes lois sur celles de la nature, et mon esprit s'est toujours tenu dans l'indépendance ${ }^{21}$.»

Cette impossibilité de réprimer le désir - car tout être humain est soumis à la loi du corps, c'est-à-dire une sensibilité aux plaisirs et donc des passions à satisfaire - montre 
que la «nature, imperturbablement sensible, cherche toujours détours et suppléments ${ }^{22}$. Usbek, légataire d'une vieille sagesse pratique, n'ignore pas la nécessité des plaisirs substitutifs pour satisfaire le penchant naturel des femmes en conseillant au Premier eunuque noir d'organiser en son absence une économie des plaisirs « innocents » :

Amuse-les par la musique, les danses, les boissons délicieuses: persuade-leur de s'assembler souvent. Si elles veulent aller à la campagne, tu peux les y mener [...]. Exhorte-les à la propreté, qui est l'image de la netteté de l'âme: parle-leur quelquefois de moi $^{23}$.

On peut toutefois douter de la capacité de ces plaisirs innocents à compenser les plaisirs absents et à garantir une stabilité sociale que menace le désordre des passions. La réponse que donne la suite des événements est sans équivoque. Si la société patriarcale met la femme au service de l'homme, la femme, privée de plaisirs et de liberté, travaille à retrouver sensualité et indépendance par des voies imprévues (lesbianisme et masturbation pour Zachi et Zéphis) et inaliénables (liberté sournoise ou affirmée pour Anaïs et Roxane). La femme travaille à retrouver tortueusement ses finalités naturelles (plaisir et liberté) écrasées sous l'institution privative du sérail. La révolte de Roxane, préfigurée déjà par celle d'Anaïs, est fondée sur la négation des lois ordinaires de la société considérées comme contraires à celles de la nature. Jusque dans la mort, Roxane, tout comme Anaïs, affirme les droits de la nature, qui sont ceux de l'accession à la libre satisfaction des désirs.

En fin de compte, la condition des femmes dans le sérail sommairement présentée dans le début du conte Ibrahim et Anaïs fonctionne comme une caricature de celle des femmes d'Usbek et de toute femme soumise à l'homme à cause des lois civiles imposées par une lecture masculine du Coran - qui peut être aussi celle des autres textes sacrés - dans une société misogyne. C'est dire que chez Montesquieu, la privation des plaisirs sensuels imposée aux femmes représente une situation contre-nature qui engendre la corruption des mœurs et la révolte intérieure ou affirmée. À travers cette critique de l'austérité que vivent les femmes, l'auteur vise non seulement la religion qui l'impose, mais implique aussi la société où les hommes exercent un pouvoir despotique sur les femmes car, selon l'analyse que fait Paul Hoffmann de la position de Montesquieu sur la polygamie, "toute relation entre l'homme et la femme est condamnable, qui réduit celle-ci à n'être que l'objet d'une jouissance sensuelle ${ }^{24}$ ». L'accession d'Anaïs au paradis après sa mort et le renouveau du sérail sous l'instigation du faux Ibrahim présentent un modèle d'organisation sociale harmonieuse qui réalise le bonheur collectif à travers une plus grande liberté accordée aux femmes dans la satisfaction de leurs désirs.

\section{La réhabilitation du plaisir des femmes}

Le conte Ibrahim et Anaïs plonge le lecteur dans un monde féerique où la mort permet à Anaïs de retrouver au paradis une vie meilleure que celle qu'elle a perdue. Elle est alors représentée dans la vie éternelle comme la maitresse active d'un sérail d'hommes dominés par les femmes. Montesquieu joue ici sur un ressort ancien du conte merveilleux: le monde inversé. Le sérail est composé d'hommes (gardés par des vieillards) dont l'unique fonction est de servir les plaisirs d'Anaïs. De ce fait, son bonheur parfait dans l'au-delà constitue une inversion de son malheur sur terre. La symétrie est également remarquable entre le vrai Ibrahim, méchant, jaloux et incapable de satisfaire sexuellement ses femmes qui sont enfermées, et le séduisant, 
aimable et vigoureux faux Ibrahim. Ce principe d'inversion permet aussi de désigner le paradis comme étant à l'opposé de ce qu'offre le monde humain. À la frustration sexuelle qui caractérise le sérail terrestre correspond donc la satisfaction des sens dans celui du paradis. Alors qu'Anaïs était une femme anonyme tout autant que ses coépouses dans le harem du vrai Ibrahim, elle règne désormais sur une cour. Les plaisirs de l'amour qui s'offrent à elle semblent répondre à « la retraite austère que son mari lui avait fait garder ${ }^{25}$ ». La symétrie entre les deux mondes dans le conte a pour but de ridiculiser la situation initiale et habituelle du despotisme domestique que fait régner le vrai Ibrahim sur ses femmes. Elle constitue également une parodie qui transforme le récit-cadre des Lettres persanes en permettant à Rica de brosser un tableau satirique de la situation des femmes dans le sérail d'Usbek.

5 En effet, le récit de Zuléma, rapporté par Rica, reprend l'histoire du sérail d'Usbek en la transformant au profit des femmes. D'ailleurs, ce conte conçoit le sérail et le paradis selon une perspective féminine imaginaire, celle de la narratrice Zuléma :

Comme les hommes qui auront bien vécu, et bien usé de l'empire qu'ils ont ici-bas sur nous, seront dans un paradis plein de beautés célestes et ravissantes, et telles que, si un mortel les avait vues, il se donnerait aussitôt la mort, dans l'impatience d'en jouir ; aussi les femmes vertueuses iront dans un lieu de délices, où elles seront enivrées d'un torrent de voluptés, avec des hommes divins qui leur seront soumis : chacune d'elles aura un sérail, dans lequel ils seront enfermés, et des eunuques, encore plus fidèles que les nôtres, pour les garder ${ }^{26}$.

Dans l'univers merveilleux du conte, le sérail est représenté à l'envers, de même que le paradis qui semble ne plus être réservé aux hommes, puisque les femmes y trouvent place. Le récit de Zuléma porte la marque de son opinion, puisqu'elle le raconte aux femmes en guise de leçon contre les « opinions injurieuses » des hommes. En narrant le même récit à son ami, Rica tente de transmettre le point de vue féminin à la conscience masculine représentée par le personnage d'Usbek et de l'inciter ainsi à comprendre la pensée des femmes.

Nous pouvons alors dire que le conte Ibrahim et Anaïs illustre ce que Linda Hutcheon définit comme une satire parodique qui «vise un objet hors du texte, mais utilise la parodie en tant que dispositif structurel pour réaliser son but correctif ${ }^{27}$ ». L'objet visé dans ce conte inséré dépasse largement la situation du sérail d'Ibrahim, et désigne un objet hors du texte, le sort malheureux des femmes et perpétuellement menacées d'une fin tragique dans le harem d'Usbek Le merveilleux s'avère le cadre propice à l'exploitation la plus efficace du dénigrement parodique. La place faite au plaisir féminin dans le paradis d'Anaïs constitue évidemment le fait majeur. Dans le sérail paradisiaque constitué d'hommes qui sont entièrement voués à la satisfaction de ses désirs, Anaiis profite de tous les plaisirs sensuels de l'amour. Montesquieu exploite longuement les procédés du roman libertin pour renforcer l'érotisme des scènes de plaisirs de l'héroïne :

Tout semblait concourir au ravissement de ses sens : elle entendait, d'un côté, une musique d'autant plus divine qu'elle était plus tendre; de l'autre, elle ne voyait que des danses de ces hommes divins, uniquement occupés à lui plaire. Cependant tant de plaisirs ne devaient servir qu'à la conduire insensiblement à des plaisirs plus grands. On la mena dans sa chambre : et, après l'avoir encore une fois déshabillée, on la porta dans un lit superbe, où deux hommes d'une beauté charmante la reçurent dans leurs bras. C'est pour lors qu'elle fut enivrée, et que ses ravissements passèrent même ses désirs ${ }^{28}$. 
Ce discours sensualiste réinterprète la nature féminine selon les seules exigences du corps et de ses désirs. Sur le plan politique, les rapports de dépendance sont ainsi inversés car c'est l'homme qui est désormais subordonné à la femme. Cette scène de volupté physique où Anaïs défaille de plaisirs entre les bras de superbes éphèbes renvoie aux procédés habituels du roman libertin que Montesquieu réutilise ou détourne pour assurer une sorte de connivence avec son lecteur. Il y a une insistance sur les plaisirs sensuels s'offrant à Anaïs qui pourraient contredire la leçon des Troglodytes ${ }^{29}$ selon laquelle la satisfaction des sens ne saurait assurer le bonheur. Cependant, nous sommes dans un univers merveilleux et fantaisiste, décrit de manière superlative et convenue pour un monde de félicités. De plus, à la première transgression, la pratique de l'amour, s'ajoute une seconde, la manière d'en parler. Anaïs ne parle que pour décrire ses plaisirs ou les renforcer :

Je suis toute hors de moi, leur disait-elle : je croirais mourir, si je n'étais sûre de mon immortalité. C'en est trop, laissez-moi; je succombe sous la violence des plaisirs. Oui, vous rendez un peu le calme à mes sens; je commence à respirer, et à revenir à moi-même. D'où vient que l'on a ôté les flambeaux? Que ne puis-je à présent considérer votre beauté divine ? que ne puis-je voir... Mais, pourquoi voir ? Vous me faites rentrer dans mes premiers transports ${ }^{30}$.

Dans un subtil calcul des plaisirs, Anaïs veut aller au-delà de l'assouvissement de ses désirs, elle veut multiplier ses sensations jusqu'à la saturation à travers le regard ${ }^{31}$ (elle reproduit la scène de voyeurisme d'Usbek décrite dans la Lettre 3). Elle ne se contente plus d'être une simple jouisseuse, elle devient spectatrice de sa propre jouissance. Le supplément de plaisir d'Anaïs réside dans ce regard dérobé du corps saisi dans la satisfaction de ses désirs. La constitution de ce théâtre de la sensualité où prime le tableau permet la mise en scène d'un espace saturé de plaisirs. L'œil perçoit, scrute et jouit tout autant que le reste du corps. Il y a là une inversion de l'attitude topique des femmes dans le roman libertin, qui s'évanouissent ${ }^{32}-$ ou plutôt feignent de s'évanouir - au moment décisif ou pendant l'acte sexuel. L'érotisme idéal de ce paradis, sans contrainte ni culpabilité, ne peut être formulé que par celle qui le vit. Une vision féminine de l'amour se trouve ainsi affirmée. Si l'on est tenté de voir cette liberté de ton dans la description de la satisfaction des sens comme un libertinage que charrierait le conte grivois, il est encore plus juste de considérer l'accession d'Anaïs aux plaisirs sexuels, comme non seulement légitime, mais aussi naturelle, nécessaire et juste. L'équilibre retrouvé par Anaïs montre à quel point l'abstinence sexuelle à laquelle elle a été contrainte sur terre par le vrai Ibrahim est contraire aux lois de la nature. $\mathrm{Si}$ cette aberration de la nature a vite été corrigée dans la vie merveilleuse au paradis céleste, où les sens reprennent tout leur pouvoir et leur assouvissement naturel, les autres femmes vivent dans une privation sur terre. D'ailleurs Anaïs va s'atteler à rétablir l'ordre de la nature dans le monde réel en envoyant un éphèbe libérer ses coépouses encore vivantes.

7 Le faux Ibrahim envoyé par Anaïs transforme le sérail traditionnel du monde terrestre en un endroit meilleur, où règnent l'égalité, la liberté et le bonheur, et où les femmes jouissent des mêmes droits et plaisirs que les hommes :

Le nouveau maître prit une conduite si opposée à celle de l'autre, qu'elle surprit tous les voisins. Il congédia tous les eunuques, rendit sa maison accessible à tout le monde: il ne voulut pas même souffrir que ses femmes se voilassent. C'était une chose singulière de les voir, dans les festins, parmi des hommes, aussi libres qu'eux $^{33}$. 
Le sérail qui émerge durant le règne du faux Ibrahim n'évoque plus un milieu oriental conservateur et autoritaire, il reflète la vision du paradis représenté par Zuléma au début du récit. Selon la conteuse, le droit d'équité est encouragé même dans la vie céleste, tout autant que le plaisir sexuel constamment évoqué, avec la venue du faux Ibrahim, sous la forme d'une allusion plaisante pour témoigner des avantages qu'il présente sur le vrai mari :

[Il] les surprend d'abord par son air doux et affable; et, bientôt après, il les surprend davantage par ses empressements, et par la rapidité de ses entreprises. [...] Enfin, le nouvel Ibrahim, resté maître du champ de bataille, se montra de plus en plus digne d'un tel choix, et se signala par des miracles jusqu'alors inconnus ${ }^{34}$.

Ce nouveau mode d'évocation de l'amour, où il est davantage question de vigueur amoureuse que de sensualité proprement dite, manifeste un retour au monde réel. Une réalité terrestre transfigurée selon le modèle idéal du paradis céleste, où le rapport homme/femme n'est plus construit sur une domination de la femme et l'étouffement de ses désirs par celle de l'homme, mais sur une amitié dont chacune des parties tire profit.

8 Le nouveau monde construit dans le conte, à travers l'expérience du renversement dans le paradis d'Anaïs et la reconfiguration du sérail terrestre d'Ibrahim, offre une solution utopique à une situation problématique. Dans un monde réel qui reste gouverné par l'homme, la narratrice Zuléma met en évidence une autre perspective, celle où la femme joue un rôle actif qui provoque le changement de l'ordre social. Cette expérience est rendue possible grâce au renversement partiel des rôles homme/femme. Les effets d'inversion ne sont pas simplement destinés à faire de ce récit un conte merveilleux et plaisant, mais un univers de revendication du droit des femmes au plaisir, de manière sérieuse, plus simple, moins conflictuelle, entre hommes et femmes - en tout cas une relation qui ne soit pas dissymétrique, en mettant les unes sous la domination des autres. La finalité de ce conte n'est plus à chercher alors dans le renversement des rôles, où la femme prend sa revanche sur l'homme en l'opprimant à son tour, mais dans une marche « vers un nouvel ordre masculin [...] mesuré ${ }^{35}$ » et plus conforme à la nature. D'ailleurs, à aucun moment, Anaïs n'abuse de son pouvoir sur les éphèbes qui sont à son service et dévoués à son plaisir dans le sérail céleste, de même que le sérail terrestre reste sous la tutelle des hommes tout en permettant aux femmes de jouir d'un peu plus de liberté. Les jalons de la stabilité et du bonheur dans la société sont posés dans le sérail reconfiguré à travers une libération des femmes, à la fois dans leur être et dans leurs désirs. Ainsi, le retour à la logique de la nature, surtout à travers un plus large accès des femmes aux plaisirs sensuels, fait retrouver à la société humaine sa fonction principale: assurer la pérennité de l'espèce humaine grâce à la reproduction. L'ouverture de ce lieu clos qu'est le sérail se fait dans un double excès de dépense et de gain :

Cependant il [le faux Ibrahim] ne se refusait aucune dépense; il dissipa avec une immense profusion les biens du jaloux, qui, de retour trois ans après des pays lointains où il avait été transporté, ne trouva plus que ses femmes et trente-six enfants $^{36}$.

Cette clôture du conte montre comment l'organisation sociale qu'est le sérail est passée d'un excès à son contraire. En effet, l'extrême continence à laquelle étaient soumises les femmes du vrai Ibrahim n'a fait que les rendre malheureuses et incapables de procréer. La transformation de ce lieu opérée par le faux Ibrahim illustre l'idée selon laquelle un certain degré de libéralité est nécessaire au bonheur des membres de toute structure sociale et à la survie de celle-ci grâce à une fécondité retrouvée (trente-six 
enfants). La précision du nombre d'enfants par femme (un enfant par an sur trois ans pour chacune des femmes) est une réponse à la lancinante question de la dénatalité dans la microsociété du sérail (celle d'Usbek tout comme celle du vrai Ibrahim) et de la dépopulation ${ }^{37}$ de la terre, dont la cause morale est à chercher dans cette économie restrictive de la libido féminine et la saturation du plaisir masculin. Le conte merveilleux oriental énonce ainsi les conditions de possibilité d'une société harmonieuse plus conforme à la nature : l'égalité de droit des hommes et femmes au désir et au plaisir sexuels par quoi l'espèce humaine se reproduit et assure sa pérennité. La satisfaction des sens à laquelle a accès la femme, au même titre que l'homme, est la principale caractéristique de l'univers merveilleux du conte Ibrahim et Anaïs qui énonce un modèle de société plus conforme à l'ordre de la nature, donc plus harmonieuse. Que ce soit le paradis céleste d'Anaïs qui fonctionne comme une utopie ou le monde réel restauré par l'éphèbe (le faux Ibrahim) envoyé par celle-ci, la rhétorique du récit reste constante : le droit des femmes aux plaisirs sensuels est doublement nécessaire à la construction d'une société idéale car il permet d'apaiser une relation conflictuelle entre les deux sexes, tout autant qu'il permet à l'espèce humaine d'assurer sa pérennité à travers une bonne natalité. Alors le monde féerique d'Anaïs, tout comme la contamination du monde réel par celui-là ont pour fonction de corriger un paradoxe du sérail - une société dominée par l'homme - en redonnant du plaisir à ces êtres de désirs que sont les femmes.

Le merveilleux exprime ainsi le refus de se résigner devant les forces régressives de la société, la volonté d'échapper à la médiocrité du quotidien, selon cette logique que Pierre Mabille indique bien dans cette question : «Pourquoi les contes animeraient-ils notre courage, notre curiosité, notre espoir, si nos parents avaient été victorieux et s'il nous suffisait de conserver leur conquête et de les imiter ${ }^{38}$ ? Il convient donc d'accueillir avec réserve toute tentative de voir, dans la condition heureuse d'Anaïs au paradis, une impossible réalisation ${ }^{39}$ de la félicité des femmes dans un monde réel déséquilibré. Ce genre d'explication escamote la nécessité de changement enseignée par le conte. En effet, l'envoi d'un éphèbe sur terre par Anaïs marque la condamnation de sa vie passée et du sort qui y est réservé aux autres femmes. Le faux Ibrahim, en exilant le vrai Ibrahim au ciel, permet aux femmes de reprendre goût à l'amour à la fois sentimental par son amabilité et physique par sa vigueur. Au contraire d'un renversement utopique de l'ordre masculin régnant sur terre, le merveilleux proclame la nécessité et la possibilité de changer le monde, de passer d'un présent malheureux à un avenir de félicité.

$10 \mathrm{Au}$ terme d'un aller-retour entre le monde réel et l'univers merveilleux du paradis céleste, de même que par le moyen d'une déconstruction et d'une proposition d'un modèle idéal, la structure sociale construite pour le seul plaisir des hommes, et sous leur forte domination, est complètement discréditée au profit d'un ordre social plus conforme à la nature parce que faisant plus de place à la liberté et à la sexualité des femmes. La principale constante dans le conte Ibrahim et Anaïs est en fait la sortie des femmes d'une situation malheureuse, le bonheur qu'elles ont à retrouver les plaisirs charnels et la possibilité qu'elles ont de s'ouvrir les portes de la félicité. Des malheurs du monde tel qu'il est aux plaisirs du monde tel qu'il devrait être, ce conte sert à démasquer la médiocrité de la condition féminine dans la société. L'infime partie du récit consacrée aux tourments des femmes en révèle leur caractère secondaire. En revanche, dans sa majeure partie, le conte représente l'aspiration à un monde sans 
déplaisirs et pourvu d'une véritable félicité ; et la forme que Montesquieu donne à cet espoir est le merveilleux.

\section{BIBLIOGRAPHIE}

\section{Bibliographie}

AUBRIT Jean-Pierre, Le conte et la nouvelle, Paris, Armand Colin, coll. « Cursus », 2002.

BECQ Annie, Lettres persanes de Montesquieu, Paris, Gallimard, coll. « Foliothèque », 1999.

BetTelheim Bruno, La Psychanalyse des contes de fées, traduit de l'américain par Th. Carlier, Paris, Robert Laffont, 1976.

BOURDIEU Pierre, Ce que parler veut dire. L'économie des échanges linguistiques [1982], Paris, Fayard, 2014.

COMBES Danièle, DAUNE-RICHARD Anne-Marie \& DEVREuX Anne-Marie, « Mais à quoi sert une épistémologie des rapports sociaux de sexe ? ", dans M.-Cl. Hurtig, M. Kail et H. Rouch (éds), Sexe et genre. De la hiérarchie entre les sexes, Paris, CNRS Éditions, 2003, p. 59-68.

DORNIER Carole (dir.), Lectures de Montesquieu. Lettres persanes, Rennes, PUR, coll. « Didact Français ", 2013.

DosSEvi Othniel, « Le conte et le merveilleux ou la promesse de l'âge d'or », Annales de la Faculté des Lettres et Sciences Humaines de Dakar, $\mathrm{n}^{\circ}$ 10, 1980, p. 101-126.

DUCRET Diane, La Chair interdite, Paris, Albin Michel, 2014.

DURAND-SENDRAIL Béatrice, « Les belles histoires : féminisme de Montesquieu dans les Lettres persanes ", Romance Notes, vol. 30, n 1, Fall 1989, p. 39-49

EHRARD Jean, L'idée de nature en France dans la première moitié du XVIII siècle [1963], Paris, Albin Michel, 1994.

FouCAult Michel, Surveiller et punir. Naissance de la prison [1975], Paris, Gallimard, 2001.

GAILLARD Aurélia, « Montesquieu et le conte oriental. L'expérimentation du renversement », Féeries [en ligne], $\mathrm{n}^{\circ} 2,2005$, mis en ligne le 21 février 2007, consulté le 30 juillet 2017. Disponible sur http://feeries.revues.org/107

GEFFRIAUD ROSSO Jeannette, Montesquieu et la féminité, Paris, Nizet, 1977.

GoLDZINK Jean, Charles-Louis de Montesquieu. Lettres persanes, Paris, PUF, coll. « Études littéraires », 1989.

GoLDzINK Jean, Montesquieu et les passions, Paris, PUF, coll. « Philosophies », 2001.

GROSRICHARD Alain, Structure du sérail. La fiction du despotisme asiatique dans l'Occident classique, Paris, Seuil, 1979. 
Guillaumin Colette, «Pratiques de pouvoir et idée de nature », Questions Féministes, $\mathrm{n}^{\circ}$ 2, février 1978, p. 5-30 et no 3, mai 1978, p. 5-28.

Hoffmann Paul, La Femme dans la pensée des Lumières, préface G. Gusdorf, Paris, Éditions Ophrys, 1977.

HUTCHEON Linda, «Ironie, satire, parodie. Une approche pragmatique de l'ironie », Poétique, $\mathrm{n}^{\circ} 46$, 1981, p. $140-155$.

JOMAND-BAUDRY Régine \& PERRIN Jean-François (dir.), Le Conte merveilleux au XVIII siècle : une poétique expérimentale, Paris, Kimé, 2002.

JUILLIARD Colette, Imaginaire et Orient. L'écriture du désir, Paris, L’Harmattan, 1996.

MABILle Pierre, Le Miroir du merveilleux, Paris, Éditions de Minuit, 1977.

MARTIN Christophe, Espaces du féminin dans le roman français du dix-huitième siècle, Oxford, Voltaire Foundation, SVEC, 2004.

MARTIN Christophe (dir.), Les Lettres persanes de Montesquieu, Paris, PUPS et Oxford, Voltaire Foundation, coll. « Vif », 2013.

PROPP Vladimir, Morphologie du conte suivi de Les transformations des contes merveilleux, et de L'étude structurale et typologie du conte, trad. M. Derrida, T. Todorov et C. Kahn, Paris, Seuil, coll. « Poétique », 1970.

STAROBINSKI Jean, L'oeil vivant. Corneille, Racine, La Bruyère, Rousseau, Stendhal, Paris, Gallimard, coll. « Tel », 2006.

VINCENT-BUFFAULT Anne, «Érotisme et pornographie au XVIII ${ }^{\mathrm{e}}$ siècle $:$ les dispositifs imaginaires du regard ", Connexions, $n^{\circ}$ 87, «Érotisme et transgressions », 2007/1, p. 97-104. Disponible sur https://www.cairn.info/revue-connexions-2007-1-page-97.htm

\section{NOTES}

1. A. Gaillard, «Montesquieu et le conte oriental. L'expérimentation du renversement », Féeries, $n^{\circ} 2,2005$, p. 2.

2. Le concept de Nature au XVIII ${ }^{\text {e }}$ siècle renvoie aux lois de la vie contre lesquelles l'homme ne peut rien: les fonctions naturelles et le plaisir qu'elles procurent (manger, dormir, etc.) ; les besoins de notre sensibilité, de notre activité (vouloir, conquérir, etc.) ; la conscience, la raison, un certain sens inné du vrai, du bien et du juste. Selon Jean Ehrard, la Nature, dans la pensée des Lumières, est «l'ensemble des lois que Dieu a établies dans le monde physique » et « les lois qui régissent la nature ne sont plus des impératifs théologiques mais des formules mathématiques ", L'idée de nature en France dans la première moitié du XVIII siècle [1963], 1994, p. 63. Montesquieu, dans De L'Esprit des lois (liv. I, chap. II), fait de l'instinct de conservation, la paix et le « désir de vivre en société » les premières lois naturelles. La nature, pour lui, c'est aussi le désir sexuel par quoi l'espèce humaine se reproduit et assure sa pérennité. Voir également Dix-huitième siècle, $\mathrm{n}^{\circ} 45$, « La Nature », 2013/1.

3. La prise de position d'Usbek dans ce débat illustre la conception sensible de l'être chez Montesquieu. «Il vaut bien mieux enlever l'esprit hors de ses réflexions, et traiter l'homme comme sensible, au lieu de le traiter comme raisonnable. L'âme, unie avec le corps, en est sans cesse tyrannisée ", dit Usbek à Rhédi (Lettre 33, Lettres persanes, éd. présentée, établie et annotée par J. Starobinski, Paris, Gallimard, 2003, p. 107). Toutes nos citations, sauf indication contraire, 
renvoient à cette édition. Consulter aussi Montesquieu, Lettres persanes, choix de lettres, lecture accompagnée par C. Volpilhac-Auger, Paris, Gallimard, coll. « La bibliothèque Gallimard », 1999.

4. Sur cette question, voir D. Ducret, La Chair interdite, 2014, chap. 2, «La pucelle à l'oreille »; P. Hoffmann, La Femme dans la pensée des Lumières, préface G. Gusdorf, 1977, chapitre liminaire, «Les spéculations théologiques sur la féminité »; J. Geffriaud Rosso, Montesquieu et la féminité, 1977 ; C. Juilliard, Imaginaire et Orient. L'écriture du désir, 1996.

5. Montesquieu, Lettres persanes, Lettre 141, éd. citée, p. 303.

6. Sur le rapprochement entre le sérail et le despotisme, voir J.Goldzink, Charles-Louis de Montesquieu. Lettres persanes, 1989, p. 71 ; A. Grosrichard, Structure du sérail. La fiction du despotisme asiatique dans l'Occident classique, 1979 ; B. Durand-Sendrail, «Les belles histoires : féminisme de Montesquieu dans les Lettres persanes ", Romance Notes, vol. 30, n 1, Fall 1989, p. 39-49.

7. Dans Surveiller et punir. Naissance de la prison [1975], 2001, Michel Foucault voit la prison comme l'instrument de pouvoir des sociétés modernes qui se sont construites sur une organisation minutieuse de la discipline. La maîtrise du corps devient ainsi un enjeu de pouvoir. Selon lui, il y a une indissociabilité du pouvoir et du corps comme sa cible privilégiée.

8. Montesquieu, Lettres persanes, Lettre 3, éd. citée, p. 53.

9. Ibid., Lettre 6, p. 59.

10. Ibid., Lettre 3, p. 55.

11. Ibid., Lettre 6, p. 59.

12. Ibid., p. 58-59.

13. Selon Jean Ehrard, le bonheur naturel dont rêvent le XvIII ${ }^{\mathrm{e}}$ siècle «n'est plus au terme d'une vie de pénitence et de mortification, mais dans la satisfaction spontanée du désir ", ouvr. cité, p. 543. Il devient ainsi impossible de construire un bonheur qui méprise les appétits naturels.

14. P. Bourdieu, Ce que parler veut dire. L'économie des échanges linguistiques [1982], 2014, p. 234. Sur l'inconscient social de cette mythologie de la dévirilisation, en rapport avec la théorie des climats, voir le chapitre III de ce livre, p. 227-239.

15. C. Martin, Espaces du féminin dans le roman français du dix-huitième siècle, SVEC, 2004, p. 348-350.

16. Sur ce sujet, voir en particulier C. Guillaumin, "Pratiques de pouvoir et idée de nature", Questions Féministes, n 2, février 1978, p. 5-30 et n 3, mai 1978, p. 5-28 ; D. Combes, A.-M. DauneRichard et A.-M. Devreux, "Mais à quoi sert une épistémologie des rapports sociaux de sexe? ", dans M.-Cl. Hurtig, M. Kail et H. Rouch (dir.), Sexe et genre. De la hiérarchie entre les sexes, 2003, p. 59-68.

17. J. Goldzink, Montesquieu et les passions, 2001, p. 12.

18. Pour Montesquieu, le droit naturel est une forme de régulation parmi d'autres, distincte à la fois du droit des gens, du droit canon et du droit civil. Loin d'être le fruit de la «droite raison », il n'est généralement que l'effet du sentiment ou de l'instinct. Alors que la conservation, la nourriture et la sexualité forment la substance de la loi naturelle.

19. Dans ce roman à la première personne publié à titre posthume en 1796 , il fait parler une jeune religieuse, Suzanne Simonin, enfermée malgré elle au couvent. À partir de cette situation (elle avait intenté un procès à sa mère pour essayer de se libérer, en prouvant qu'elle avait été contrainte), Diderot imagine la vie qu'elle a menée dans trois couvents successifs. Il fait ainsi une satire impressionnante des couvents de femmes au XVIII ${ }^{\mathrm{e}}$ siècle. Cette claustration contre-nature développe chez les religieuses des comportements malsains (brimades mesquines allant jusqu'au sadisme, perversions sexuelles), dans un climat d'hypocrisie et de haine qui les conduit parfois à la folie. Diderot montre ainsi, selon les réflexions qu'il prête à Sœur Suzanne, que «l'homme est né pour la société ; [et que] [...] la retraite déprave ».

20. Montesquieu, Lettres persanes, Lettre 4, éd. citée, p. 55.

21. Ibid., Lettre 161, p. 340.

22. J. Goldzink, ouvr. cité, p. 9.

23. Montesquieu, Lettres persanes, Lettre 2, éd. citée, p. 53. 
24. P. Hoffmann, ouvr. cité, p. 335.

25. Montesquieu, Lettres persanes, Lettre 141, éd. citée, p. 307.

26. Ibid., p. 303.

27. L. Hutcheon, «Ironie, satire, parodie. Une approche pragmatique de l'ironie », Poétique, n 46, 1981, p. 148

28. Montesquieu, Lettres persanes, Lettre 141, éd. citée, p. 304-305.

29. L'histoire des Troglodytes (Lettres 11 à 14) est conçue comme une réponse à la question «si les hommes [sont] heureux par les plaisirs et les satisfactions des sens, ou par la pratique de la vertu » (Lettre 10).

30. Montesquieu, Lettres persanes, Lettre 141, éd. citée, p. 305.

31. Sur la prééminence du regard dans l'analyse des passions et de la littérature érotique, voir A. Vincent-Buffault, «Érotisme et pornographie au XVIII ${ }^{\mathrm{e}}$ siècle : les dispositifs imaginaires du regard ", Connexions, n 87, 2007/1, p.97-104; J. Starobinski, L'œil vivant. Corneille, Racine, La Bruyère, Rousseau, Stendhal, 2006, p. 9-28.

32. La perte de contrôle total de soi ou l'évanouissement, avec la notion de "petite mort» comme métaphore de la jouissance, est une image que l'on rencontrera dans le roman libertin comme Les Liaisons dangereuses (1782) de Laclos (évanouissement de Mme de Tourvel dans les bras de son séducteur Valmont, Lettre 125) et La Religieuse de Diderot (perte de connaissance de la supérieure dans les bras de Suzanne).

33. Ibid., p. 310.

34. Ibid., p. 308.

35. A. Gaillard, art. cité, p. 9.

36. Montesquieu, Lettres persanes, Lettre 141, éd. citée, p. 310.

37. Voulant apaiser les inquiétudes du jeune Rhédi sur la dépopulation du globe et sur la décadence (Lettre 112), Usbek rédige une dissertation à dimension planétaire. Si l'on excepte les épidémies et les catastrophes naturelles, quelles sont les causes morales, religieuses, politiques et économiques de la dépopulation? Usbek fait alors le procès des religions dominatrices dont l'esprit de querelle use l'énergie des peuples (Lettre 119). À l'opposé, le commerce et l'esprit d'entreprise jouent en faveur de la poussée démographique. Il faut donc supprimer ce qui est improductif : le célibat des prêtres ou le droit d'aînesse qui force les cadets à entrer dans l'armée ou en religion (Lettres 117 et 119). Usbek dénonce également l'esclavagisme qui anéantit la vie de milliers d'hommes pour exploiter l'or et l'argent (Lettre 118). Il examine ensuite comment la polygamie orientale, l'existence d'eunuques et l'avortement freinent la procréation (Lettres 114 et 120); comment, à l'inverse, le divorce l'encourage (Lettre 116). Enfin, il s'en prend au colonialisme tout entier qui, par esprit de profit à court terme, gaspille les ressources naturelles et humaines de la planète (Lettre 121). Seuls les régimes politiques modérés trouvent grâce aux yeux d'Usbek: ils favorisent la paix civile, l'initiative, une meilleure répartition des biens et l'esprit familial, facteurs d'essor démographique. A contrario, les régimes despotiques étouffent l'esprit civique et le progrès (Lettres 122 et 123).

38. P. Mabille, Le Miroir du merveilleux, 1977, p. 54.

39. L'analyse d'Aurélia Gaillard va dans ce sens quand elle affirme que "la "fable" orientale inventée par Montesquieu renonce, in extremis, à l'utopie pour enseigner les conditions de nonpossibilité » dans " Montesquieu et le conte oriental. L'expérimentation du renversement », art. cité, p. 9. 


\section{RÉSUMÉS}

La question du plaisir des femmes se pose avec acuité dans les Lettres persanes de Montesquieu, surtout dans le conte merveilleux inséré dans la lettre 141 qu'est l'histoire d'Ibrahim et Anaïs. L'objectif de cet article est de montrer comment l'univers sensible du conte merveilleux Ibrahim et Anaïs participe à la réflexion sur la gestion du plaisir féminin dans les sociétés souvent dominées par les hommes et donne une réponse à cette équation. Si dans les sociétés patriarcales la femme est condamnée à ne satisfaire que les désirs de l'homme, le conte Ibrahim et Anaïs fait de la réhabilitation du plaisir féminin la condition d'une société harmonieuse et pérenne. Ce conte merveilleux semble donc montrer comment la répression du désir féminin devient un facteur de déséquilibre pour la société et la libération de la sensualité féminine la solution pour une harmonie sociale.

The issue of women's pleasure arises with acuteness in the Lettres persanes by Montesquieu, particularly in the supernatural story within the letter 141 that is the story of Ibrahim and Anaïs. The aim of this article is to show how the sensitive universe of the supernatural story "Ibrahim et Anaïs" participates in the reflection on the treatment of female pleasure in societies mostly dominated by men and how it also gives an answer to that equation. If in chauvinist societies women are condemned to satisfy men's desires, the story "Ibrahim et Anaïs" makes the rehabilitation of female pleasure the condition for a harmonious and lasting society. Thus, this supernatural tale seems to show how the repression of female desire becomes a factor of imbalance for society and how the release of female sensuality becomes the solution for social harmony.

INDEX

Keywords : tales, supernatural, desire, pleasure, woman, sensuality, Montesquieu Mots-clés : conte, merveilleux, désir, plaisir, femme, sensualité, Montesquieu

\section{AUTEUR}

\section{MOUSSA TRAORÉ}

Université Clermont Auvergne, IHRIM 